\title{
Comparative study of Metallocene catalyst propylene polymerization with different iteration rates
}

\author{
Subita Bhagat *1, Nikhil Prakash 2 \\ 1 Assistant Professor, Department of Chemical Engineering, SLIET-Longowal, 148106, India. \\ 2 Assistant Professor, Department of Chemical Engineering, SLIET-Longowal, 148106, India. \\ Corresponding Author Email: subitabhagat424@gmail.com
}

\begin{abstract}
This paper proposed a mathematical model corresponding to metallocene catalyzed propylene polymerization that uses the Me2Si [Ind]2ZrCL2 and Et [Ind]2ZrCL2. Comprehensive kinetic models consisting of mass and population balance equations, are developed based on elementary reactions proposed in the reaction mechanism. The result from the above indicates that metallocene catalysts in the presence of ethylene zirconium dichloride and methylene zirconium dichloride shows modularity and new peaks are obtained. The temperature variation from 25 to 75 also increase the rate and reason for the same could be chain shuttling polymerization. The model is presented through simulative study. Initially genetic approach is used but convergence rate is poor. To achieve best possible result, particle swarm optimization is used. The optimization approach with particle swarm optimization is implemented. The local and global solutions are comparable entities and replace each other in case value of local variable is not optimized. From the simulative study it is discovered that Et [Ind]2ZrCL2 produce best possible polymers both at 25 and $750 C$.
\end{abstract}

Keywords: Metallocene catalyst, Kinetic models, ethylene zirconium

\section{Introduction}

The utilization of polypropylene with the propylene generally is inexpensive and critical research that leads to the stereo block polymerization. [1]This is generally a part of crosslinking networks that may retain elastomeric properties. [2] These properties include more stability and toughness. Because of these properties, it is commonly used as the additives for buildings, highways etc. Me2Si [Ind]2ZrCL2 and Et [Ind]2ZrCL2 as a metallocene catalyst in presence of similar conditions shows similar results but in the dissimilar conditions new peaks have been attained. [3] They fall into asymmetric homogeneous catalyst. [4]The microstructure of polypropylene generally depends upon the structure of catalyst symmetry along with the secondary catalyst involved.

In the proposed work, binary catalyst system is used different proportion of stereoselectivity. To achieve the optimal transfer of contents between two metals, chain shuttling polymerization is employed. This mechanism is useful in attaining the black 
polyolefin. The same procedure is implemented in the proposed work to check the feasibility study.

[5]This study is based upon the optimization strategies that could be used especially within simulative study. [6] The optimization strategy initially employed within the proposed work is genetic algorithm. [7]The temperature and rate constants are varied corresponding to each iteration. The convergence rate however is poor. To avoid the situation, particle swarm optimization is used.

The smaller molecules within the catalyst will serve as particles. The particles will be selected randomly. [8], [9]Afterword's local and global solutions obtained will be stored within the variables. [10]The optimization result obtained will be retained.[11] The global solution in the first iteration will serve as local solution in the next iteration. Mutation and crossover will be the next phase. The mutation will be used to generate the next offspring and best possible selection will be obtained through the crossover phase.

\section{Materials and Methods Employed}

\section{- Used Material}

The manipulation within the proposed work is carried out in the argon atmosphere.[12] Me2Si [Ind]2ZrCL2 and Et [Ind]2ZrCL2 was synthesized through the variation of temperature from 25 to 75 0C. [13] The MAO in toluene is provided by the labs in Bathinda. The polymerization was purified by applying distinct temperatures and filtering mechanism. Nitrogen based atmosphere is used for refluxing toluene.

\section{- Polymerization}

This is the process in which large number of monomer molecules react together to form a polymer. The produced structure after polymerization in the proposed work is branched. [14]This means no linear structure is produced in this case. Free radical reactions are one of the most common ways of producing polymers. This mechanism in the presence of Me2Si [Ind]2ZrCL2 and Et [Ind]2ZrCL2 is used to produced branch polymers. [15]The temperature is varied to evaporate solvent to obtain a polymer at fast rate. New peaks with the presence of Me2Si [Ind]2ZrCL2 and Et [Ind]2ZrCL2 is obtained. Table 1 indicates the result after applying proposed process

Table 1: Propylene polymerization through Me2Si[Ind]2ZrCL2 and Et[Ind]2ZrCL2

\begin{tabular}{|c|c|c|}
\hline $\mathbf{T}^{\mathbf{O}} \mathrm{C}$ & 25 & 75 \\
\hline$k_{i n} \times 10^{3} \quad\left(\mathbf{M}^{-1} \cdot \mathbf{s}^{-1}\right)$ & 7.870525 & 15.74105 \\
\hline$k \times 10^{-4}{ }_{P}^{\left(\mathbf{M}^{-1} \cdot \mathrm{s}^{-1}\right)}$ & 4.172725 & 8.34545 \\
\hline$k_{d} \times 10^{4} \quad\left(\mathrm{~s}^{-1}\right)$ & 3.029002 & 6.058004 \\
\hline$\left(M^{-1} \cdot s^{-1}\right)$ & 4.003503 & 8.007006 \\
\hline $\begin{array}{r}K b \times \mathbf{1 0}^{\mathbf{6}} \\
, H\end{array}$ & 2.525051 & 5.050102 \\
\hline 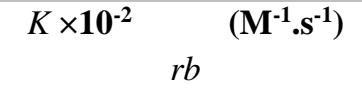 & 3.631215 & 7.26243 \\
\hline
\end{tabular}




\begin{tabular}{|c|c|c|}
\hline$\left(M^{-1} \cdot s^{-1}\right)$ & 3.434840 & 6.86968 \\
\hline $\begin{array}{c}k \quad \times 10^{4} \quad\left(\mathbf{M}^{-1} \cdot \mathbf{s}^{-1}\right) \\
s p\end{array}$ & 1.712346 & 3.424692 \\
\hline$\left(M^{-1} \cdot s^{-1}\right)$ & 7.908342 & 15.816684 \\
\hline$K t, A l \quad\left(\mathbf{M}^{-1} \cdot \mathbf{s}^{-1}\right)$ & 5.838210 & 11.67642 \\
\hline$\left(M^{-1} \cdot s^{-1}\right)$ & 1.155187 & 2.310374 \\
\hline$F(k)$ & 1000 & - \\
\hline
\end{tabular}

The polymerization condition includes setting up the time from 0 to 30 minutes. Temperature variation from 25 to $750 \mathrm{C}$.

\section{Result and performance analysis}

The experiment of propylene polymerization is conducted in the presence of Me2Si[Ind]2ZrCL2 and Et[Ind]2ZrCL2. The experiment can be used to design the optimal binary catalyst system. [16] The result is based on kinetic model. The performance of the catalyst is shown within table 1. [17] The chain mutation is used to achieve the new peaks. The used Methodology for optimization is given in figure 1

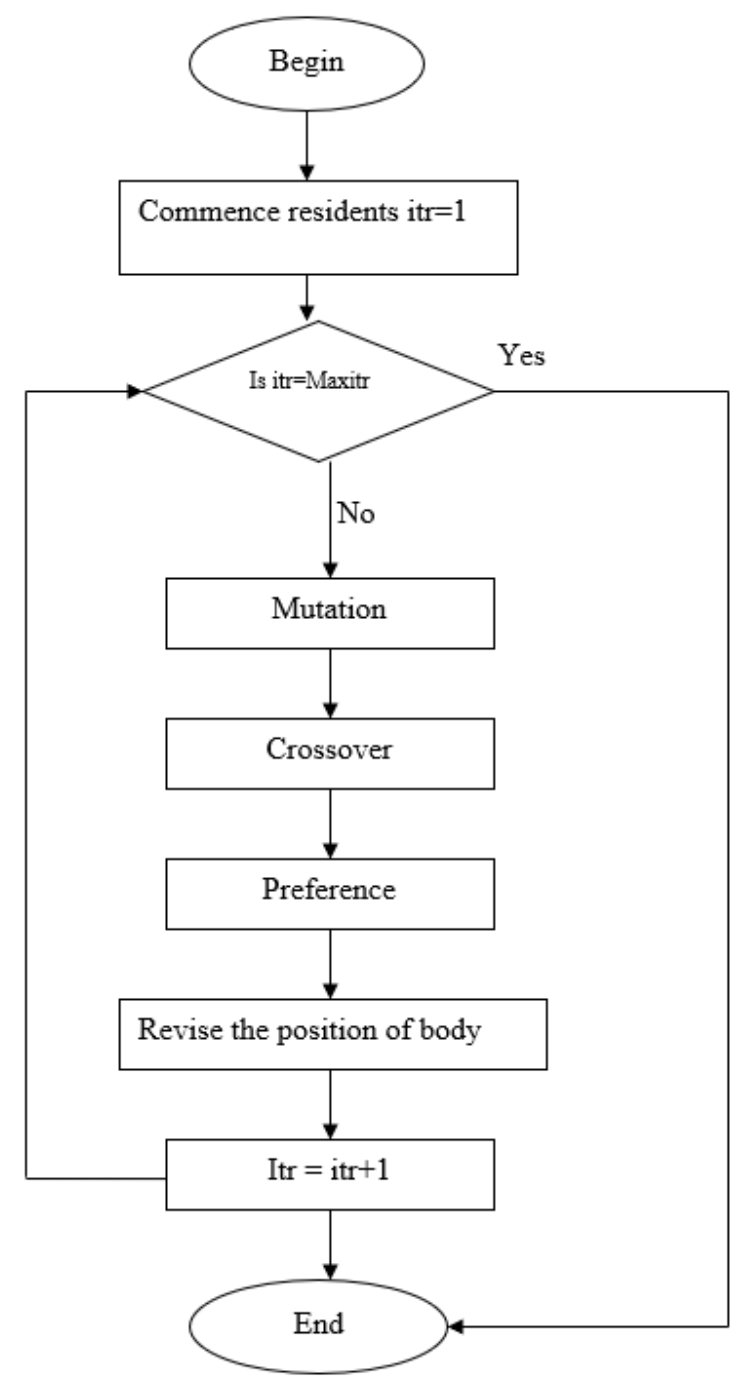

Figure 1 Proposed Methodology for obtaining the solid polymer using Me2Si [Ind]2ZrCL2 and Et [Ind]2ZrCL2 
The population selection is the initial phase in the attainment of polymer using the propylene polymerization in the presence of Me2Si [Ind]2ZrCL2 and Et [Ind]2ZrCL2. The monomer molecules are randomly deployed and selected in the initial phase. After the selection of monomers, fixed set of iterations are set. The global and local solution is obtained. The global solution is retained, and mutation phase is applied. The mutation phase is used to generate offspring by combining monomer polymers. The crossover phase will decide the monomer selection for generating the polymer. In case global solution is better as compared to the local solution, then global solution will be replaced with the local solution. The applied procedure generates new peak when analyzed under similar conditions. The iterative approach followed will converge only if the result is below the threshold levels. The mechanism follows the threshold levels of 0.2. in case obtained global values of 0.2 , the process converges. The polymerization obtain gives the better result and higher peaks as compared to mechanism without the use of Me2Si [Ind]2ZrCL2 and Et[Ind]2ZrCL2.The result for polymerization with Me2Si[Ind]2ZrCL2 and $\mathrm{Et}[\mathrm{Ind}] 2 \mathrm{ZrCL} 2$ is given in figure 2

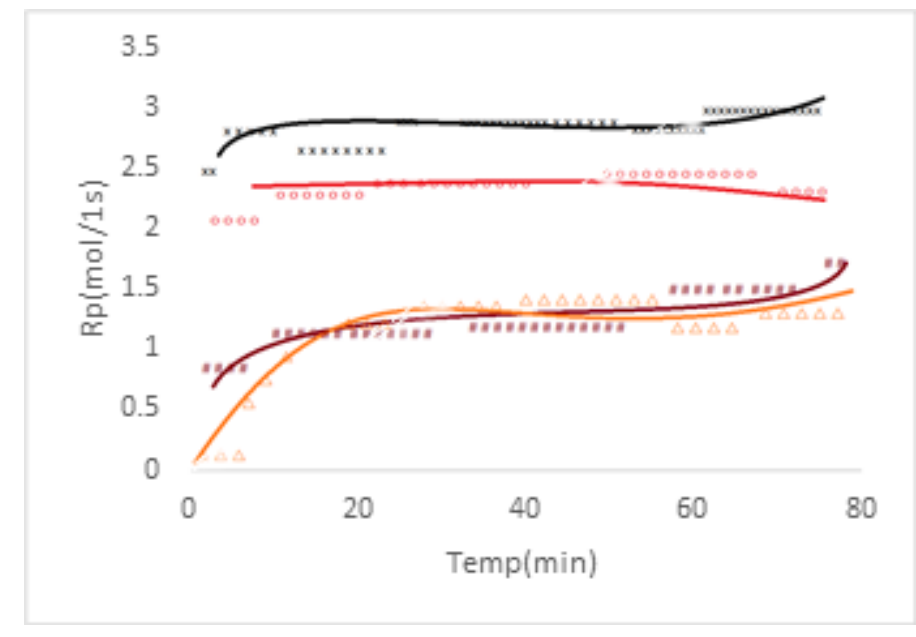

Figure 2 Result of Polymerization with Me2Si [Ind]2ZrCL2 and Et [Ind]2ZrCL2 using 50 iterations and reaction time of 30 minutes

' $\mathrm{x}$ ' indicates the $750 \mathrm{C} / 2000(\mathrm{Me} 2 \mathrm{Si}$ [Ind]2ZrCL2)

' $\mathrm{o}$ ' indicates the $75 \mathrm{oC} / 500$ (Et [Ind]2ZrCL2)

' $\#$ ' indicates the $25 \mathrm{oC} / 2000(\mathrm{Me} 2 \mathrm{Si}$ [Ind]2ZrCL2)

' $\triangle$ ' indicates the $25 \mathrm{oC} / 500$ (Et [Ind]2ZrCL2)

The obtained result shows Me2Si [Ind]2ZrCL2 and Et [Ind]2ZrCL2 deviation but positively towards the new peak. This also indicates that polymers will be obtained with high accuracy. The iteration in the first case is limited but in the second case, polymerization is conducted with 100 iterations. 


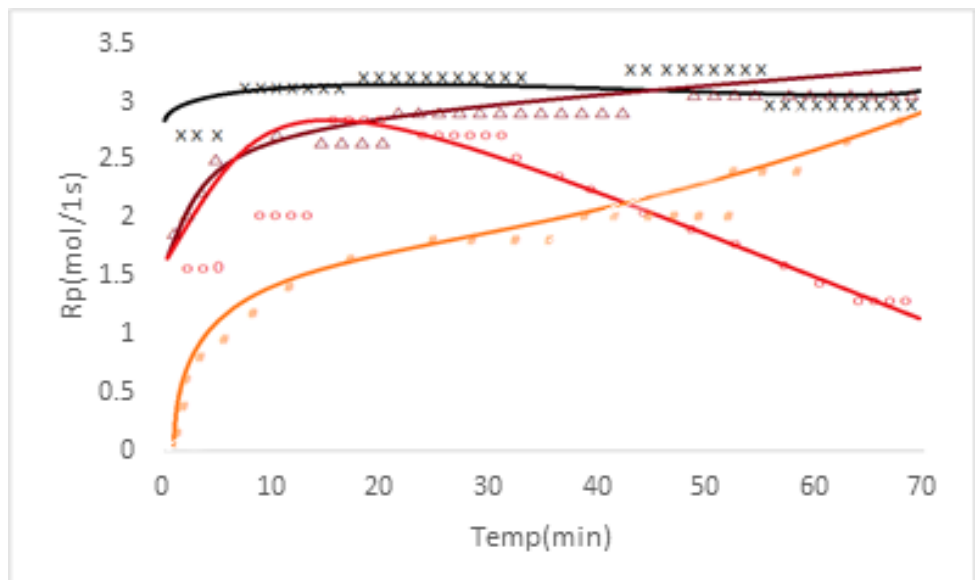

Figure 3 Polymerization with Me2Si [Ind]2ZrCL2 and Et [Ind]2ZrCL2 at 25 and 750C with 100 iterations

' $\mathrm{x}$ ' indicates the $750 \mathrm{C} / 2000(\mathrm{Me} 2 \mathrm{Si}$ [Ind]2ZrCL2)

' $\mathrm{o}$ ' indicates the $75 \mathrm{oC} / 500$ (Et [Ind]2ZrCL2)

'\#' indicates the $25 \mathrm{oC} / 2000(\mathrm{Me} 2 \mathrm{Si}$ [Ind]2ZrCL2)

' $\triangle$ ' indicates the $25 \mathrm{oC} / 500$ (Et [Ind]2ZrCL2)

At 250C, the downfall of polymerization with Me2Si [Ind]2ZrCL2 takes place. This indicates that result is not in an optimal zone and generated polymers may not be tough. To resolve the issue, iterations are increased, and time interval is kept at the constant values of 30 minutes. Both the results for Me2Si [Ind]2ZrCL2 and Et [Ind]2ZrCL2 are comparable but at 50 iterations result is much better as compared to 100 iterations.

To validate the result, number of iterations are again varied from 100 to 150 . The issue of deviation again appears for Me2Si [Ind]2ZrCL2. The variation indicates generated polymers may not be stable. The most effective result thus is obtained for the iterations 50. The mutation is set to 0.3 in this case and more solid polymers are obtained. Overall, the result at 150 iterations, result obtained is given within figure 3 . The temperature at which showing maximum deviation of propylene polymerization with $\mathrm{Me} 2 \mathrm{Si}$ [Ind]2ZrCL2 and Et [Ind]2ZrCL2 is 25.

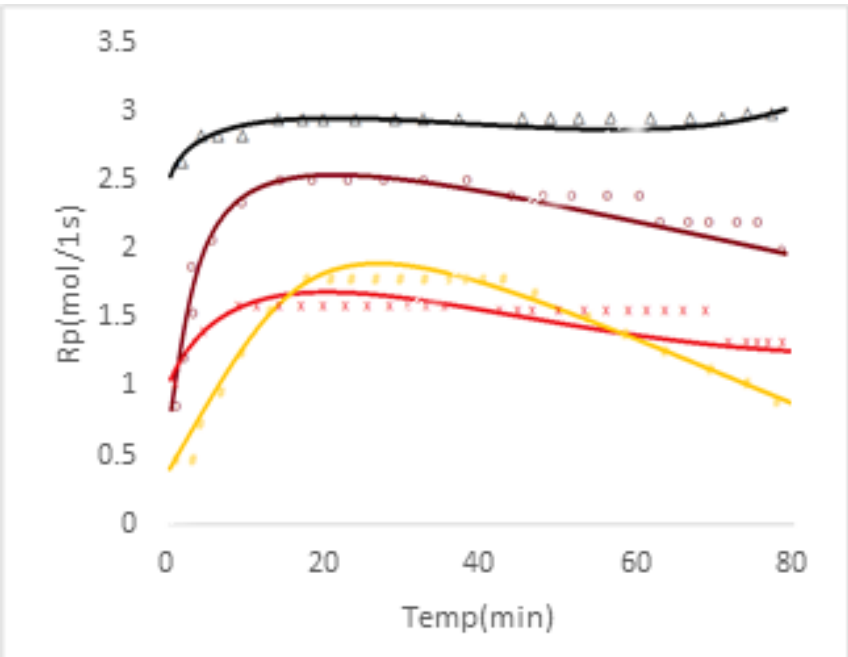

Figure 4 Propylene polymerization with $\mathrm{Me}_{2} \mathrm{Si}$ [Ind $]_{2} \mathrm{ZrCL}_{2}$ and $\mathrm{Et}$ [Ind $]_{2} \mathrm{ZrCL}_{2}$ at 25 and $75^{\circ} \mathrm{C}$ 
' $\mathrm{x}$ ' indicates the $750 \mathrm{C} / 2000(\mathrm{Me} 2 \mathrm{Si}[\mathrm{Ind}] 2 \mathrm{ZrCL} 2)$

' $\mathrm{o}$ ' indicates the $75 \mathrm{oC} / 500$ (Et [Ind]2ZrCL2)

'\#' indicates the $25 \mathrm{oC} / 2000(\mathrm{Me} 2 \mathrm{Si}$ [Ind]2ZrCL2)

' $\triangle$ ' indicates the $25 \mathrm{oC} / 500$ (Et [Ind]2ZrCL2)

\section{Conclusion}

The metallocene catalyst with Me2Si [Ind]2ZrCL2 and Et [Ind]2ZrCL2 are evaluated by varying the temperature from $250 \mathrm{C}$ to $750 \mathrm{C}$. The temperature range varied to determine the rate of generation of polymers. It was discovered that at $750 \mathrm{C}$ best possible result is obtained with Et [Ind]2ZrCL2. The Me2Si [Ind]2ZrCL2 shows inconsistencies at 250C. The iterations are varied from 50 to 150 and it was discovered that curve is downgraded indicating weak formation of polymer with Me2Si [Ind]2ZrCL2. The result can be improved by increasing the concentration of Me2Si [Ind]2ZrCL2 propylene. To conclude, it is discovered that Et [Ind]2ZrCL2 generates more stable polymer as compared to Me2Si [Ind]2ZrCL2.

\section{References}

[1] A. Ali et al., "Rapid kinetic evaluation of homogeneous single-site metallocene catalysts and cyclic diene: how do the catalytic activity, molecular weight, and diene incorporation rate of olefins affect each other?," RSC Advances, vol. 11, no. 50, pp. 31817-31826, 2021, doi: 10.1039/D1RA06243C.

[2] F. C. Franceschini, T. T. D. R. Tavares, J. H. Z. dos Santos, M. L. Ferreira, and J. B. $P$. Soares, "Ethylene and propylene polymerization using in situ supported Me 2Si(Ind)2ZrCl2 Catalyst: Experimental and theoretical study," Macromolecular Materials and Engineering, vol. 291, no. 3, pp. 279-287, 2006, doi: 10.1002/MAME.200500360.

[3] L. Resconi, L. Cavallo, A. Fait, and F. Piemontesi, "Selectivity in propene polymerization with metallocene catalysts," Chemical Reviews, vol. 100, no. 4, pp. 1253-1345, Apr. 2000, doi: 10.1021/CR9804691.

[4] K. S. Prakash, "(600y) Kinetic Modeling of Propylene Polymerization with Me2Si[Ind]2ZrCl2/MAO Catalyst System | AIChE,” AICHE ACADEMY, 2014. https://www.aiche.org/conferences/aiche-annual-

meeting/2014/proceeding/paper/600y-kinetic-modeling-propylene-polymerizationme2siind2zrcl2mao-catalyst-system-1 (accessed Dec. 11, 2021).

[5] C. R. Reeves, "A genetic algorithm for flowshop sequencing," Computers \& Operations Research, vol. 22, no. 1, pp. 5-13, Jan. 1995, doi: 10.1016/03050548(93)E0014-K.

[6] S. K. Singh and D. P. Vidyarthi, "Independent tasks scheduling using parallel PSO in multiprocessor systems," International Journal of Grid and High Performance Computing, vol. 7, no. 2, pp. 1-17, 2015, doi: 10.4018/IJGHPC.2015040101.

[7] Oshin and M. C. Bhatt, "Hybrid PSO for job scheduling to minimize makespan in heterogeneous grids," in Proceedings of the 2nd International Conference on 
Communication and Electronics Systems, ICCES 2017, Mar. 2018, vol. 2018January, pp. 586-591. doi: 10.1109/CESYS.2017.8321145.

[8] R. Qayyum, K. Kamal, T. Zafar, and S. Mathavan, "Wood Defects Classification Using GLCM Based Features And PSO Trained Neural Network,” IEEE ACCESS, pp. 3-7.

[9] R. K. Jena, "Multi objective Task Scheduling in Cloud Environment Using Nested PSO Framework,” Procedia - Procedia Computer Science, vol. 57, pp. 1219-1227, 2015, doi: 10.1016/j.procs.2015.07.419.

[10] W. Abdulal, A. Jabas, S. Ramachandram, and O. al Jadaan, "Task Scheduling in Grid Environment Using Simulated Annealing and Genetic Algorithm," 2012.

[11] P. Murali, A. Sandur, and A. A. Patil, "Correction of Logical Errors in $\{C\}$ programs using Genetic Algorithm Techniques," International Journal of Recent Trends in Engineering (IJRTE), vol. 1, no. 2, pp. 176-178, 2009, [Online]. Available: http://ijrte.academypublisher.com/vol01/no02/ijrte0102176178.pdf

[12]W. Kaminsky, "ANNALS OF ADVANCES IN CHEMISTRY Polyolefi $n$ nanocomposites based on metallocene catalysts," hspc, 2021, doi: 10.29328/journal.aac.1001022.

[13] S. Jungllng, R. Mulhaupt, U. Stehllng, / Hans-Herbert Brintzinger, D. Fischer, and F. Langhauser, "Propene Polymerization Using Homogeneous MAO-Activated Metallocene Catalysts: Me2Si(Benz[e]lndenyI)2ZrClu'MAO vs. Me2Si(2-MeBenz[e]lndenyI)2ZrClu'MAO," Erich in. Journal of Polymer, 1995, doi: 10.1002/pola.1995.080330813.

[14] T. Sun, L. Wang, X. Dong, W. Wang, and L. I. Wang, "An insight into the chainpropagation mechanism of propylene polymerization catalyzed by traditional Tibased Ziegler-Natta catalysts in view of recently developed catalysts," Designed Monomers and Polymers, vol. 9, no. 2, pp. 117-127, 2006, doi: 10.1163/156855506776382691.

[15] F. C. Franceschini, T. T. da R. Tavares, J. H. Z. dos Santos, J. B. P. Soares, and M. L. Ferreira, "Comparative study of propylene polymerization using Me2Si(RInd)2ZrCl2/SiO2-SMAO/AlR3 and Me2Si(RInd)2ZrCl2/MAO (R=Me, H), " Polymer, vol. 48, no. 7, pp. 1940-1953, Mar. 2007, doi: 10.1016/J.POLYMER.2007.01.062.

[16] F. C. Franceschini, T. T. da R. Tavares, J. H. Z. dos Santos, J. B. P. Soares, and M. L. Ferreira, "Comparative study of propylene polymerization using Me2Si (RInd )2ZrCl2 /SiO2-SMAO/AlR3 and Me2Si(RInd)2ZrCl2/MAO (R = Me, H)," undefined, vol. 48, no. 7, pp. 1940-1953, Mar. 2007, doi: 10.1016/J.POLYMER.2007.01.062.

[17] P. S. Kulyabin et al., "Ansa-Zirconocene Catalysts for Isotactic-Selective Propene Polymerization at High Temperature: A Long Story Finds a Happy Ending," Journal of the American Chemical Society, vol. 143, no. 20, pp. 7641-7647, May 2021, doi: 10.1021/JACS.1C03380/SUPPL_FILE/JA1C03380_SI_002.XYZ. 